\title{
Propuesta para conciliar el trabajo y la familia en una compañía del sector automotriz
}

DOI: https://doi.org/10.33262/ap.v3i3.64

Propose to reconcile the work and the family in a company of the automotive sector

Katherine Lorena Sanguña Carrera. ${ }^{1}$ \& Washington Edy Santillán Marroquín. ${ }^{2}$

\begin{abstract}
.
In order to obtain an authentic conciliation between the work and the familiar life it is required of a great dose of discipline, planning and organization in both systems (laborrelative) so that a imbalance does not exist that affects on the one hand, psiquis of the worker and by another one, the productivity in its place of work, we suggested some directives and directions to them to obtain this intention that takes us to the investigation. Objective. To generate good labor and familiar practices, using tools practical to obtain conciliation and quality of life in the work and the families of the workers of the company. Methodology. The present project has a descriptive methodology and analytical, which it generates quantitative and qualitative information, which looks for "to specify the properties, the characteristics and the profiles of people, groups, communities, processes, objects or any other phenomenon related to the subject, that is to say, they measure, they evaluate or they collect data on diverse concepts (variable), aspects, dimensions or components of the phenomenon to investigate". Results. The final sample was conformed by 35 people who toil in a automotive company. The workers have a high degree of schooling, a $17.50 \%$ consider that they have a flexible schedule, finish to their labor day $51 \%$ of the workers between 18:30 and 19:00 hours, around 90\% of the personnel he thinks that he is due to separate the

\footnotetext{
${ }^{1}$ Magister en Gestión del Talento Humano, Universidad Internacional SEK, ksanguna@hotmail.com ORCID: https://orcid.org/0000-0003-2625- 815X/print, Quito- Ecuador.

${ }^{2}$ Coordinador Académico del Instituto Superior Tecnológico Bernardo O'Higgins, coordinacion.academica@instituto-ohiggins.com ORCID: https://orcid.org/0000-0003-1980- 6832, Quito- Ecuador.
} 
subjects of work and the familiar life; finally, the company promotes some practices to foment this conciliation between the work and the familiar life. Conclusion. The practices that would have to improve in the company go focused to the flexibility of schedules, teletrabajo, remunerations, race plans, next to the opportune vacations in time and permissions to take care of subjects familiar.

Key words: quality of life, labor conciliation, practices, work, family, results.

\section{Resumen.}

Para lograr una auténtica conciliación entre el trabajo y la vida familiar se requiere de una gran dosis de disciplina, planificación y organización en los dos sistemas (laboralfamiliar) para que no exista un desequilibrio que afecte por un lado, la psiquis del trabajador y por otro, la productividad en su lugar de trabajo, les sugerimos algunas directrices y orientaciones para lograr este propósito que nos lleva a la investigación.

Objetivo. Generar buenas prácticas, laborales y familiares utilizando herramientas prácticas para lograr conciliación y calidad de vida en el trabajo y en las familias de los trabajadores de la compañía. Metodología. El presente proyecto tiene una metodología descriptiva y analítica, que genera información cuantitativa y cualitativa, el cual busca "especificar las propiedades, las características y los perfiles de personas, grupos, comunidades, procesos, objetos o cualquier otro fenómeno relacionado con el tema, es decir, miden, evalúan o recolectan datos sobre diversos conceptos (variables), aspectos, dimensiones o componentes del fenómeno a investigar". Resultados. La muestra final estuvo conformada por 35 personas que laboran en una empresa automotriz. Los trabajadores tienen un alto grado de escolaridad, un 17,50\% consideran que tienen un horario flexible, terminan su jornada laboral el 51\% de los trabajadores entre las 18:30 y 19:00 horas, alrededor del 90\% del personal cree que se debe separar los temas de trabajo y la vida familiar;finalmente, la empresa promueve algunas prácticas para fomentar dicha conciliación entre el trabajo y la vida familiar. Conclusión. Las prácticas que debería mejorarse en la empresa van enfocadas a la flexibilidad de horarios, teletrabajo, remuneraciones, planes de carrera, junto a las vacaciones a tiempo y permisos oportunos para atender temas familiares.

Palabras Claves: calidad de vida, conciliación, prácticas laborales, trabajo, familia, resultados.

\section{Introducción.}

El concepto de equilibrio entre la vida laboral y familiar en las últimas décadas ha crecido de manera acelerada por la importancia que tiene el talento humano en las empresas y su valoración en la calidad de vida. En las organizaciones, lo que se pretende es generar cambios en los aspectos: humanos, económicos, ambientales y sociales, enfocados en relacionar la cultura empresarial junto a las competencias y el potencial del negocio para apoyar a cada uno de sus equipos de trabajo, para conseguir 
un balance entre las funciones que desempeñan dentro de sus horas laborales y el tiempo que dedican a su contexto familiar.

Los entes gubernamentales aportan con leyes especializadas del trabajo para que los trabajadores generen condiciones óptimas para que puedan ejercer sus funciones tanto laborales como responsabilidades familiares que logren equilibrio. Ejemplo el teletrabajo hoy tan difundido en la pandemia. Otro ejemplo, la protección de la maternidad, esto genera una percepción que el rol femenino es apoyado y protegido.

Teniendo en cuenta la información anterior, se buscó diseñar un programa de equilibrio entre la vida laboral y familiar para los trabajadores de una empresa automotriz ubicada en la ciudad de Quito, la cual se ha dedicado a la venta de automóviles por más de 50 años.

\section{Metodologia.}

Este proyecto de investigación tiene una metodología descriptiva y analítica, que genera información cuantitativa y cualitativa, el cual busca "especificar las propiedades, las características y los perfiles de personas trabajadoras, grupos, comunidades, procesos, objetos o cualquier otro fenómeno relacionado con el tema; es decir, miden, evalúan o recolectan datos sobre diversos conceptos (variables), aspectos, dimensiones o componentes del fenómeno a investigar" (Hernández, Fernández y Baptista, 2006, p.104).

Para lo cual se determinó un período específico para su realización, dentro de este esquema, se empezó con una charla enfocada a la explicación y socialización sobre el tema de la "conciliación de la vida familiar y personal" de los trabajadores que laboran en una empresa automotriz, ubicada en la ciudad de Quito, en donde se obtuvo los principales criterios para estructurar el cuestionario del tema.

El enfoque destinado para este proyecto es de tipo cuantitativo, dado que se "utiliza la recolección y análisis de datos para contestar preguntas de investigación y probar hipótesis establecidas previamente" (Gómez, 2006, p. 60). En el caso de esta investigación se utilizó un cuestionario, que sirvió para la recolección de datos.

\section{Resultados.}

La muestra está conformada por el personal que labora en una empresa automotriz ubicada en la ciudad de Quito, que cumple con las siguientes características: género, tanto femenino como masculino; escolaridad, bachiller, pregrado y postgrado; además, pertenece al área administrativa, distribuida de la siguiente manera: presidencia, gerencia general, recursos humanos, financiero, contabilidad, cartera, tesorería, importaciones, sistemas, marketing, calidad, servicios generales y repuestos.

La variables socio-demográficas, fueron enfatizadas, debido a que se estableció una diferenciación de los elementos en variables como: género, edad, instrucción académica y cargos que ocupan. 
Tabla 1

Criterios de inclusión y exclusión de la muestra.

\begin{tabular}{cc}
\hline Criterios de inclusión & Criterios de exclusión \\
\hline $\begin{array}{c}\text { Participantes de ambos sexos. } \\
\text { Tener formación académica mínima } \\
\text { de bachiller. }\end{array}$ & $-\begin{array}{c}\text { Personal con discapacidad alta } \\
\text { diagnosticada en la parte cognitiva. }\end{array}$ \\
$-\quad \begin{array}{l}\text { Personal que ocupen cargos } \\
\text { administrativos en la empresa } \\
\text { automotriz. }\end{array}$ & $-\begin{array}{c}\text { Personal que se encuentren en las } \\
\text { áreas técnicas, postventa y ventas. }\end{array}$ \\
- Permanencia en el cargo designado \\
por un periodo no menor a 3 meses.
\end{tabular}

Tabla 1. Alfa publicaciones

Fuente: Elaboración propia.

\section{Recolección de datos}

Para la recolección de datos se usó un cuestionario dividido en 12 preguntas en donde los participantes basados en su situación laboral actual responderán acorde a su criterio, dichas preguntas fueron construidas a través de la información obtenida en las charlas explicativas sobre el tema.

Las preguntas 1, 2, 9 y 10 están conformadas por preguntas en donde se debe escoger una opción que se asemeje a la situación actual. Las preguntas 3, 4, 5, 6, 7, 8, 11 están conformadas por preguntas en la escala de Likert como se plantea en la siguiente tabla:

Tabla 2

Escalas de Likert

\begin{tabular}{ll}
\hline \multicolumn{1}{c}{ Por Acuerdo } & \multicolumn{1}{c}{ Por frecuencia } \\
\hline Totalmente de acuerdo & Muy frecuente \\
De acuerdo & Frecuentemente \\
Neutral & Ocasionalmente \\
En desacuerdo & Raramente \\
Totalmente en desacuerdo & Nunca \\
\hline
\end{tabular}

Tabla 2. Alfa publicaciones

Fuente: Elaboración propia.

La pregunta 13 está conformada por varios criterios que pueden elegir los participantes de la muestra.

Las encuestas fueron enviadas a través de un link a los correos electrónicos del personal que cumplía con los criterios de inclusión establecidos.

\section{Procedimiento}

1. Para obtener la aprobación y realizar este proyecto, se contactó con la Gerencia General de la empresa, se realizó una reunión explicativa del proyecto y se logró 
inmediatamente la aceptación del mismo y más bien el ejecutivo y la empresa se mostraron optimistas en colaborar con esta investigación.

2. Una vez otorgada la autorización, se procedió a fijar una fecha y hora para explicar al grupo de participantes varias inquietudes que servirían para la creación del cuestionario. Así mismo, para tener un mejor conocimiento sobre la parte sociodemográfica de la empresa se requirió datos de género, edad, escolaridad y puesto que ocupan para un posterior análisis.

3. Para la explicación del tema se dividió en dos grupos con horarios diferentes para no dejar los puestos inactivos. Una vez coordinado y explicado el tema a investigar se procedió a explicar que el cuestionario sería enviado a cada correo eléctrico personal con las siguientes instrucciones:

El siguiente cuestionario tiene como finalidad el conocer el "equilibrio de la vida laboral y familiar" que existe en la empresa.

Resulta importante mencionar que la información obtenida será utilizada con fines académicos, investigativos y estrictamente confidenciales.

\section{Instrucciones:}

"Sea lo más honesto posible en sus respuestas. Para completar el cuestionario seleccione la casilla correspondiente a la respuesta que más se acerque a su realidad."

4. Una vez recolectada toda la información del instrumento, se procedió a ingresar dicha información en el programa de Excel ejecutando el análisis respectivo.

\section{Plan de análisis de datos}

Una vez recolectados los datos obtenidos con el instrumento y el procesamiento de los mismos, se inició el procesamiento de la información por medio de un análisis estadístico, en donde se emplearon programas tales como:

1. Microsoft Office Excel

2. A su vez se aplicó estadística descriptiva que permitió describir los datos y puntuaciones obtenidas, las técnicas estadísticas que se utilizaran son:

\section{Medidas de tendencia central: media.}

3. Para cada una de las variables a medir, se utilizaron barras de gráficos.

La muestra final estuvo conformada por 35 personas que laboran en una empresa automotriz del área administrativa ubicada en la ciudad de Quito.

\section{Datos demográficos}

Los datos demográficos obtenidos son los siguientes: 
Gráfico 3

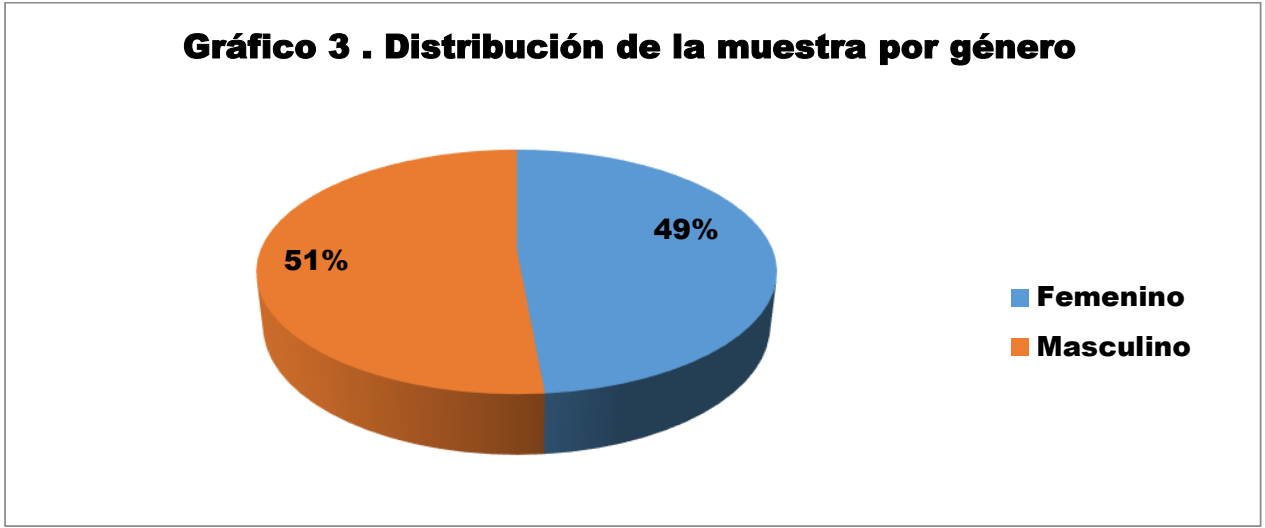

Gráfico 3. Alfa publicaciones

Fuente: Elaboración propia.

Como se observa en el gráfico de las 35 personas encuestadas, 18 corresponden al género masculino con un 51\% mientras que 17 corresponden al género femenino con un $49 \%$. Resaltando que en este giro de negocio existe un equilibrio en la contratación del personal tanto masculino como femenino.

\section{Gráfico 4}

Gráfico 4. Distribución de la muestra por nivel de instrucción

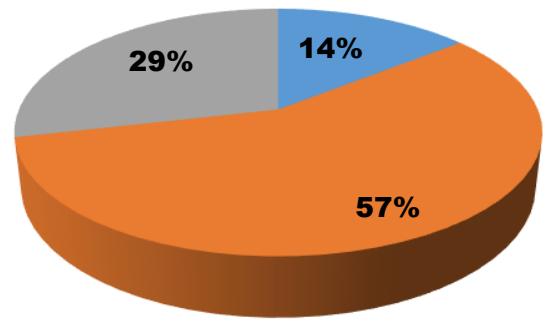

Bachiller

Pregrado

Postgrado

\section{Gráfico 4. Alfa publicaciones}

Fuente: Elaboración propia.

Al observar el gráfico del grado de instrucción, se evidencia que un 57\% del personal presenta título de pregrado, donde un $29 \%$ del personal posee un título de postgrado mientras que un $14 \%$ posee un título de bachiller, lo cual muestra, que la empresa automotriz tiene personal con un alto grado de escolaridad. 


\section{Gráfico 5}

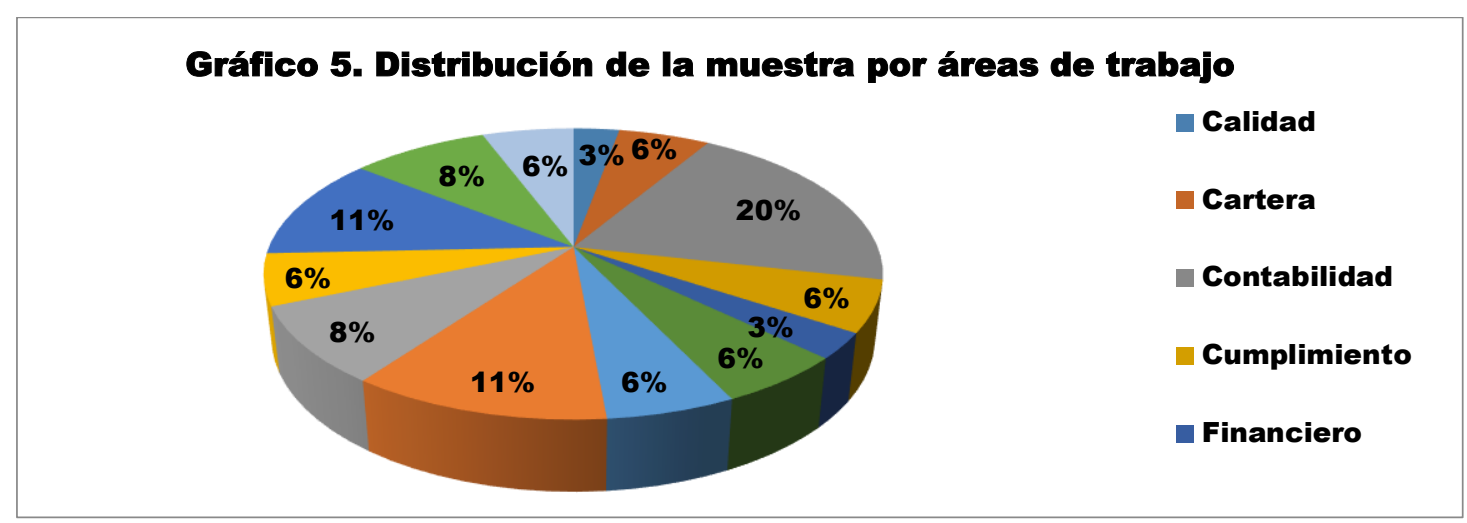

Gráfico 5. Alfa publicaciones

Fuente: Elaboración propia.

Como representa el gráfico, el área con más número de personas que la conforman es contabilidad con el $20 \%$, con el $11 \%$ se encuentran las áreas de marketing como repuestos contrastando con las áreas de calidad como financiera que tienen el 3\% de la muestra del total del personal que labora en la empresa.

\section{Encuesta sobre conciliación de la vida laboral y familiar}

A continuación, se mostrarán los resultados de las preguntas formuladas al personal del área administrativa que labora dentro de una empresa automotriz:

Para el análisis de resultados se procedió a sacar la frecuencia, se realizó un conteo de cuantas personas llenaron la encuesta tomando en consideración las preguntas con escala de Likert tanto por acuerdo como frecuencia así como las opciones de selección múltiple.

\section{Pregunta 1:}

\section{Gráfico 6}

¿Tiene usted un horario flexible de trabajo?

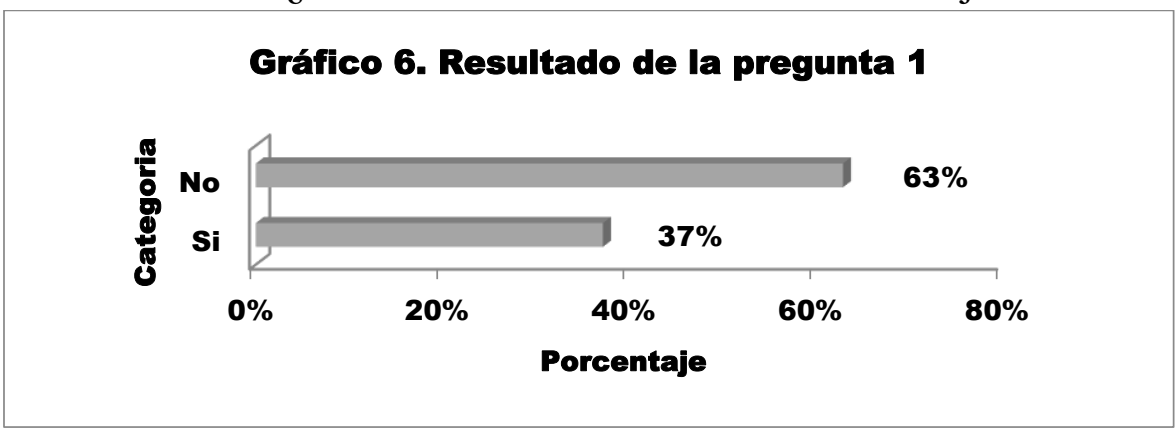

Gráfico 6. Alfa publicaciones

Fuente: Elaboración propia.

Del total de la muestra se puede determinar que el $63 \%$ que es equivalente a 22 personas están de acuerdo con tener un horario flexible de trabajo, mientras que el $43 \%$ equivalente a 13 personas no creen tener flexibilidad laboral. 
Por lo cual, se puede mencionar que más de la mitad de los encuestados no creen tener un horario flexible.

\section{Pregunta 2}

\section{Gráfico 7}

¿A qué hora termina habitualmente su jornada laboral?

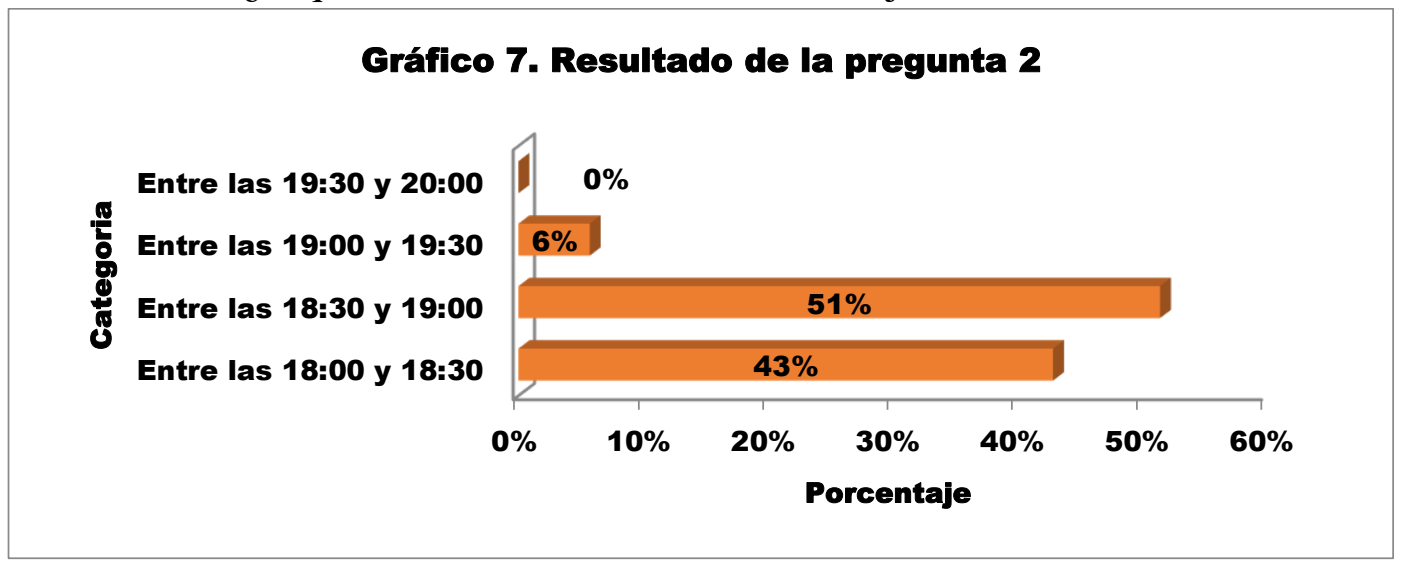

Gráfico 7. Alfa publicaciones

Fuente: Elaboración propia.

El gráfico muestra los horarios en los que las personas encuestadas terminan sus jornadas laborales, presentado un $51 \%$ equivalente a 18 personas que salen entre las $18 \mathrm{~h} 30$ a $19 \mathrm{~h} 00$, un $43 \%$ equivalente a 15 personas cumplen con los horarios de $18 \mathrm{~h} 00$ a $18 \mathrm{~h} 30$, con un porcentaje de $6 \%$ equivalente a 2 personas terminan su jornada entre las $19 \mathrm{~h} 00$ a $20 \mathrm{~h} 00$.

Por lo tanto, se puede mencionar que si se agrupan las respuestas con mayor porcentaje nos daría que el $94 \%$ de los encuestados representado por 33 personas reconocen que terminan sus funciones más tarde de las horas establecidas en sus contratos de trabajo.

\section{Pregunta 3}

Gráfico 8

¿Con que frecuencia prolonga la jornada laboral semanalmente?

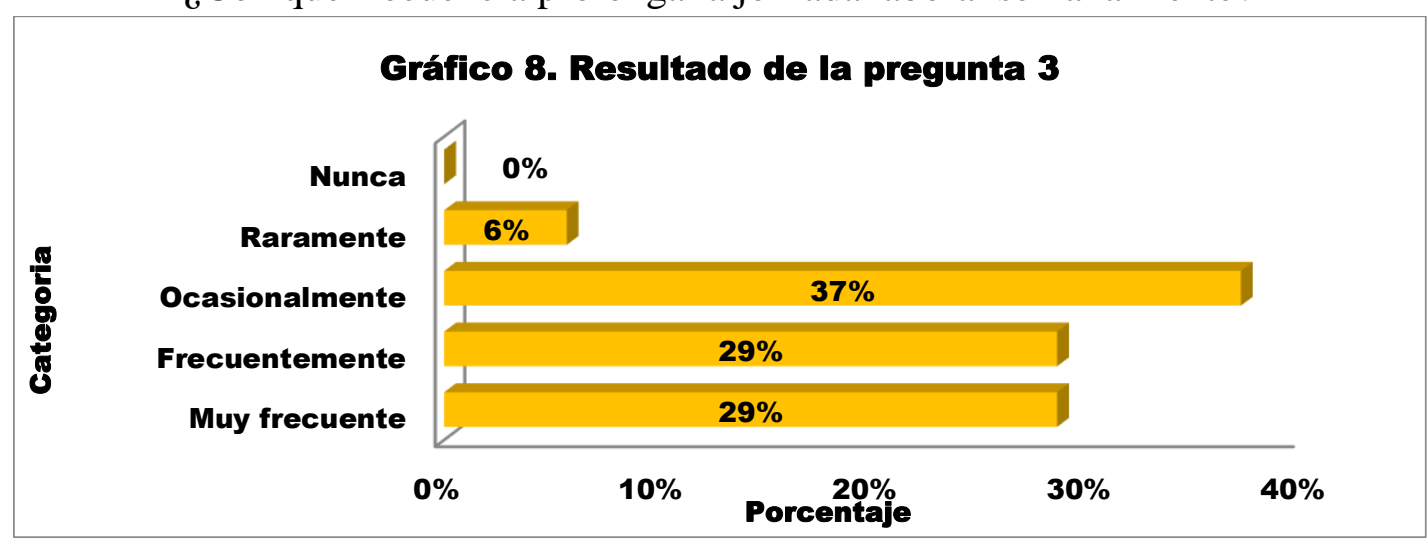

Gráfico 8. Alfa publicaciones

Fuente: Elaboración propia. 
Se puede acotar de los resultados de este gráfico, que el $37 \%$ que es el equivalente a 13 personas ocasionalmente prolongan sus jornadas laborales, el $29 \%$ equivalente a 10 personas respectivamente creen quedarse más tiempo frecuentemente, mientras que solo el $6 \%$ equivalente a 2 personas admiten que raramente se quedan laborando fuera de sus horas establecidas para ejercer sus funciones.

\section{Pregunta 4}

\section{Gráfico 9}

¿Cree usted que la empresa considera al trabajo más importante que la familia?

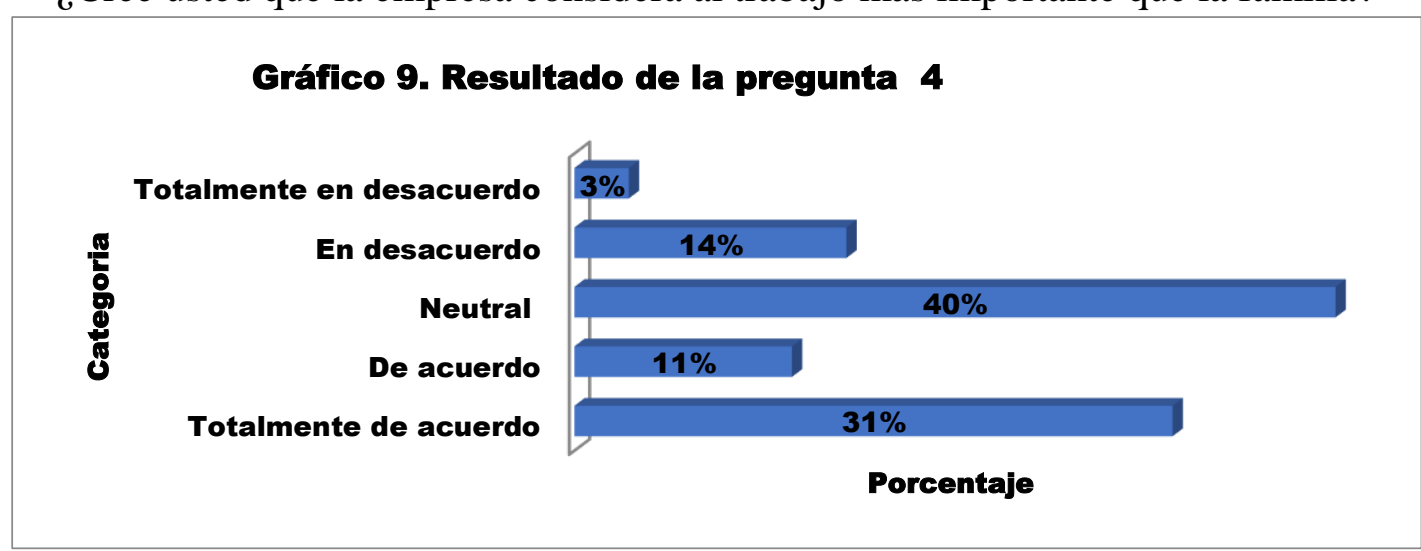

\section{Gráfico 9. Alfa publicaciones}

Fuente: Elaboración propia.

De los resultados de la muestra obtenidos en este gráfico, se puede observar que el $31 \%$ conformado por 11 personas se encuentran totalmente de acuerdo con este índice, el $11 \%$ conformado por 5 personas se encuentran de acuerdo, el $40 \%$ conformado por 12 personas se muestran indiferentes a estas preguntas, el 14\% representado por 6 personas de los encuestados se muestran en desacuerdo y el $3 \%$ representado por 1 persona se encuentra totalmente en desacuerdo con este índice.

\section{Pregunta 5}

\section{Gráfico 10}

¿Usted está de acuerdo con la siguiente afirmación: "Hay que separar la vida personal de la laboral?

\section{Gráfico 10. Resultado de la pregunta 5}

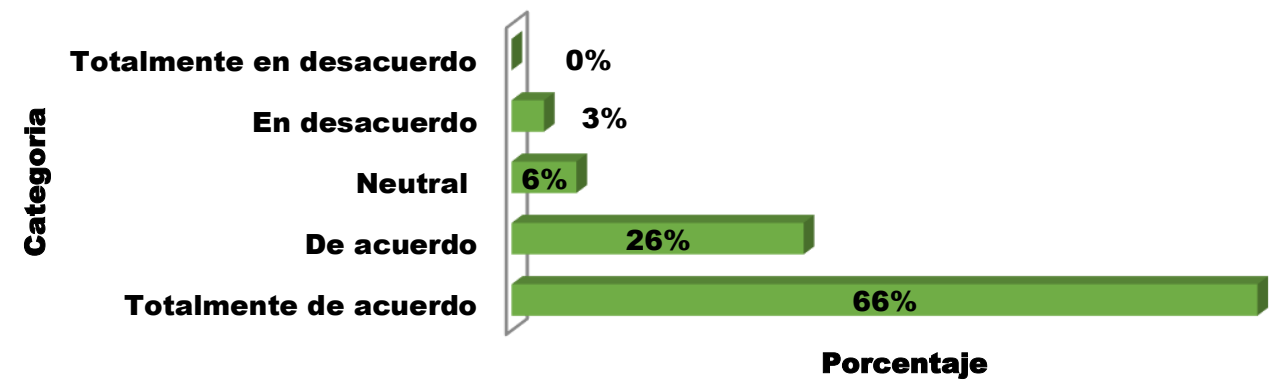

Gráfico 10. Alfa publicaciones

Fuente: Elaboración propia. 
En la pregunta sobre separar el tema laboral de la vida personal la muestra respondió con 12 personas conformando un $66 \%$ se encuentran totalmente de acuerdo, el $26 \%$ conformada por 9 personas se mostraron de acuerdo con esta premisa, el $6 \%$ conformada por 2 personas se mostraron neutrales mientras que el 3\% de la muestra conformada por 1 personas se encuentran en desacuerdo.

\section{Pregunta 6}

\section{Gráfico 11}

¿Las actividades de su trabajo afectan a su vida familiar?

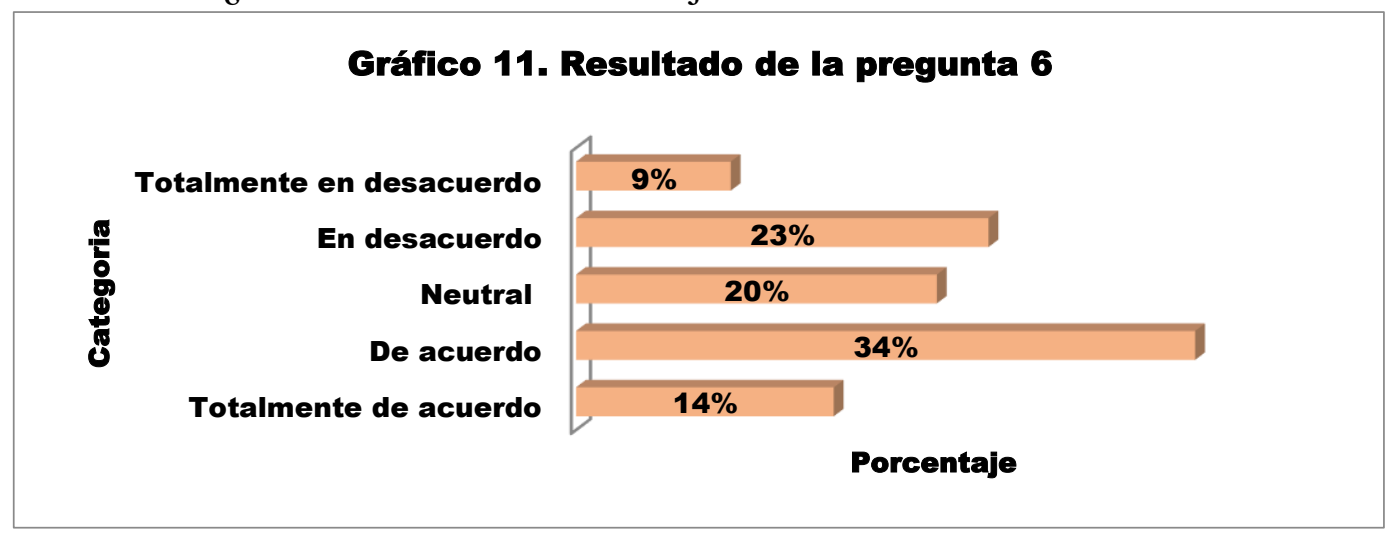

\section{Gráfico 11. Alfa publicaciones \\ Fuente: Elaboración propia.}

Los resultados muestran que el $34 \%$ conformado por 12 personas se encuentran de acuerdo, el $23 \%$ conformado por 8 personas se encuentran en desacuerdo, entretanto el $20 \%$ representado por 7 personas se muestran neutrales con la pregunta; el $14 \%$ conformada por 5 personas se muestran totalmente de acuerdo y el $9 \%$ conformada por 3 personas se encuentran totalmente en desacuerdo con la pregunta de si el trabajo ha afectado a la vida familiar de cada persona.

\section{Pregunta 7}

\section{Gráfico 12}

¿El tiempo que requiere para cumplir sus funciones en el trabajo hace que sea complicado satisfacer sus tareas familiares?

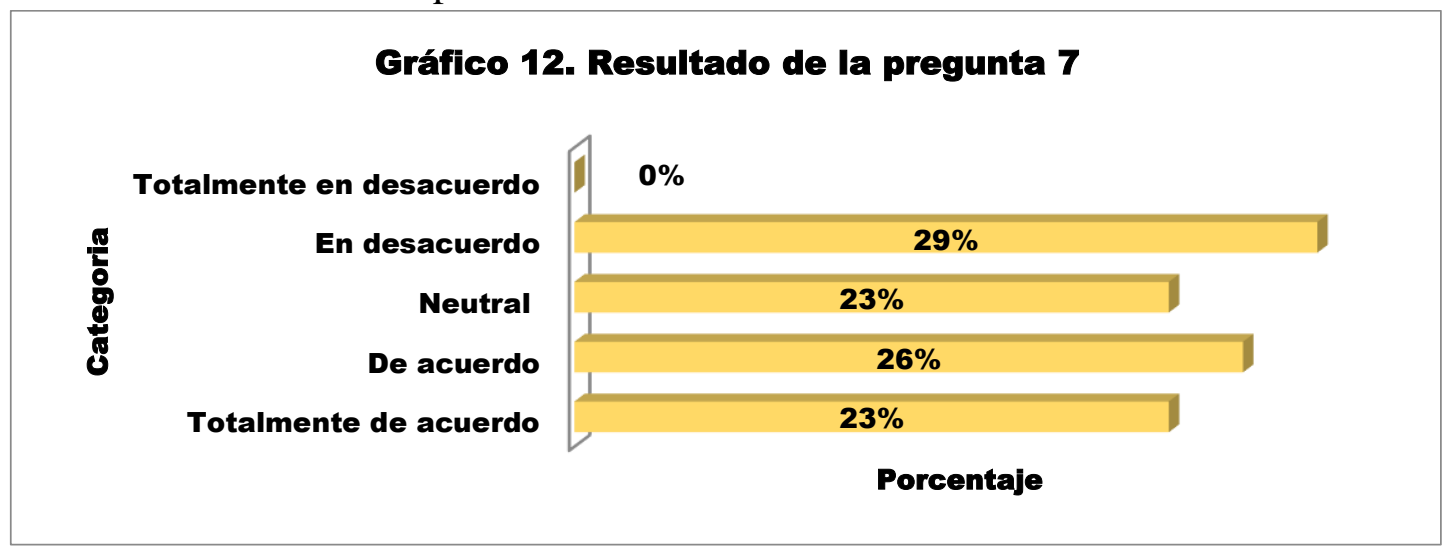

Gráfico 12. Alfa publicaciones

Fuente: Elaboración propia. 
Se puede manifestar que en la pregunta sobre si al cumplir sus tareas familiares, el trabajo se hace complicado de realizar, la muestra respondió de manera pareja, con un $29 \%$ representado por un total de 10 personas se encuentran en desacuerdo, el $26 \%$ representado por un total de 9 personas se encuentran de acuerdo con las premisas, entretanto que el $23 \%$ equivalente a 17 personas se muestran indiferentes y neutrales a este índice. Esto nos muestra que la experiencia de cada persona es diferente, por lo cual esta pregunta se la debe explorar más para tener más información para comparar.

\section{Pregunta 8}

\section{Gráfico 13}

¿Considera que las funciones que desempeña en su trabajo van acorde a su formación?

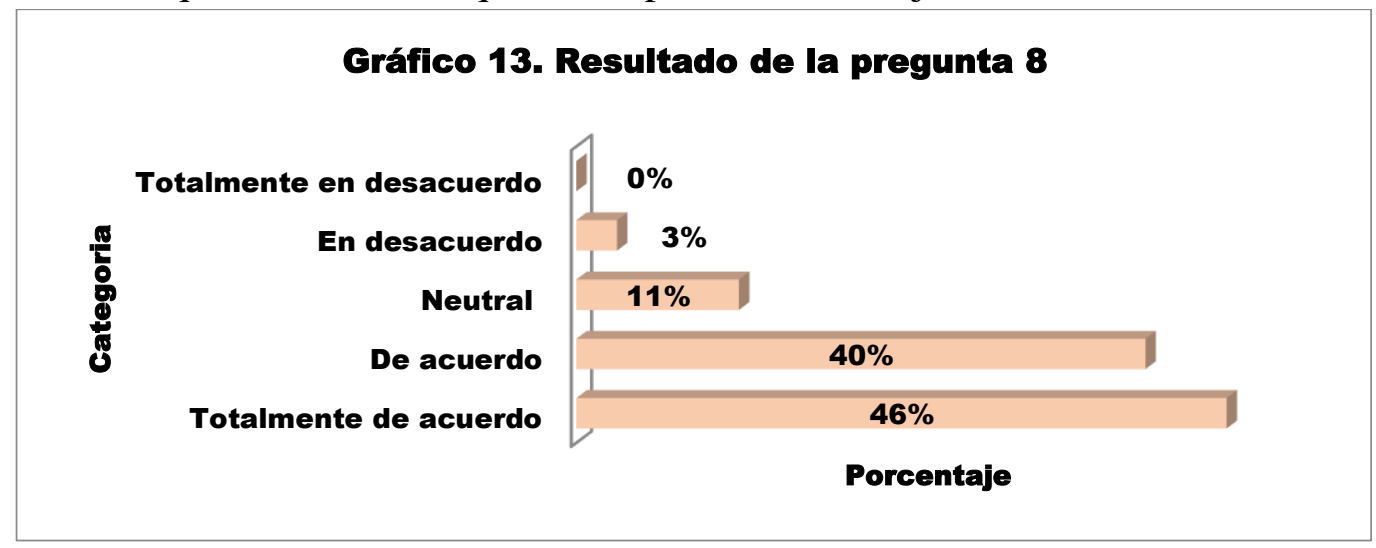

\section{Gráfico 13. Alfa publicaciones \\ Fuente: Elaboración propia.}

De las personas encuestadas se obtuvo con un $46 \%$ representado por 16 personas se encuentran totalmente de acuerdo, el $40 \%$ representado por un total de 14 personas se hallan de acuerdo, entretanto el $11 \%$ representado por 4 personas se muestran neutrales con las premisas. Sin embargo, solo el 3\% equivalente a 1 persona se muestra en desacuerdo con la premisa de que las funciones que tiene en la empresa van relacionadas con las áreas de formación académica.

\section{Pregunta 9}

\section{Gráfico 14}

¿Quién se encarga del cuidado de su/s hijos durante el tiempo que usted se encuentra en el trabajo?

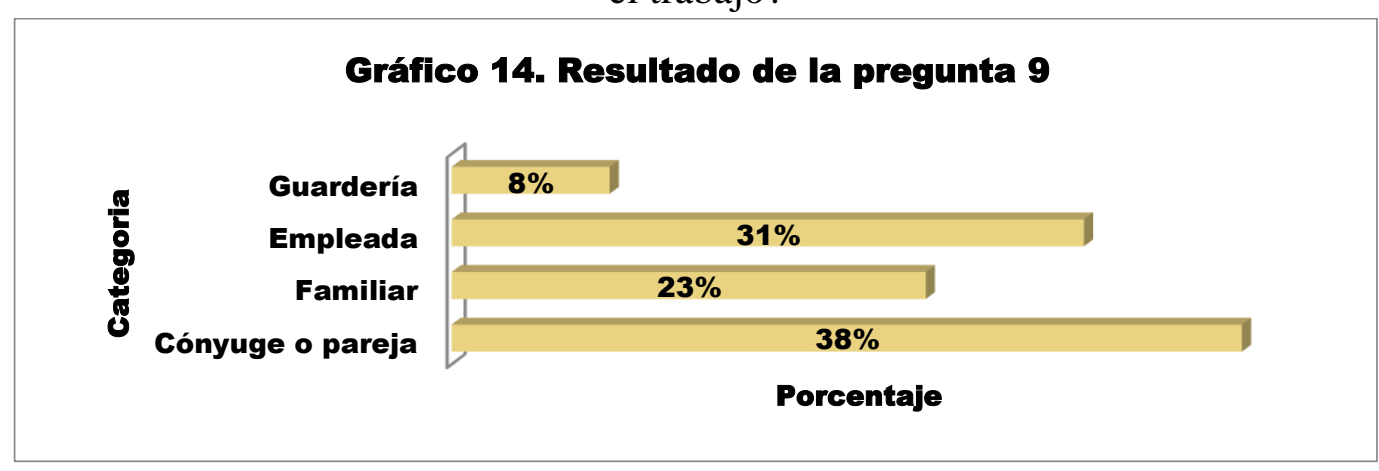

Gráfico 14. Alfa publicaciones

Fuente: Elaboración propia. 
Esta pregunta fue respondida por 13 personas que tienen hijos menores de 15 años, los cuales respondieron a la pregunta sobre quien cuida a los menores durante el tiempo que las personas que trabajan en la empresa están laborando, se puede manifestar que un $38 \%$ representado por 5 personas tienen el apoyo del cónyuge o pareja, el $31 \%$ de la muestra representado por un total de 4 personas encargan el cuidado de los hijos a la empleada doméstica, el $23 \%$ equivalente a 3 personas pide la ayuda de un familiar. Estos valores contrastan con un $8 \%$ equivalente a 1 persona que deja todo el cuidado a la guardería a la que inscribió al menor.

\section{Pregunta 10}

\section{Gráfico 15}

¿Quién se encarga del cuidado de su/s hijos durante el tiempo que tienen citas médicas, reuniones o no asisten a clases?

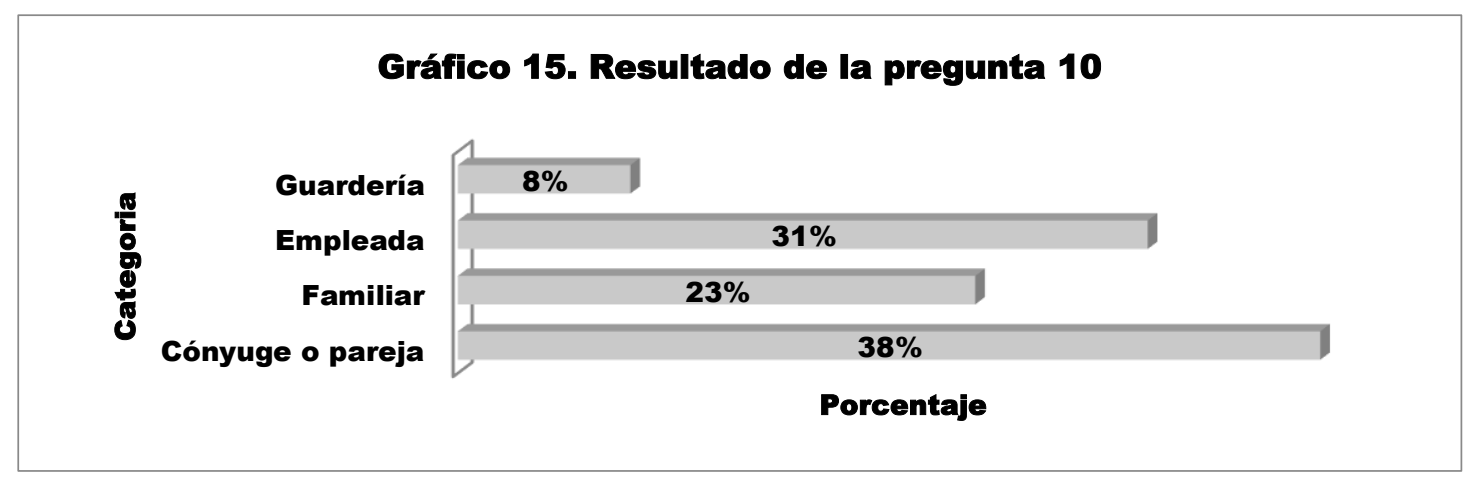

\section{Gráfico 15. Alfa publicaciones \\ Fuente: Elaboración propia.}

Al igual que la pregunta anterior solo fue respondida por 13 personas que tienen hijos menores de 15 años, para lo cual se obtuvo con un 38\% equivalente a 5 personas encargan el cuidado de sus hijos a su cónyuge o pareja, el $31 \%$ equivalente a 4 personas deja el cuidado a la empleada doméstica, a su vez el $23 \%$ equivalente a 3 personas están de acuerdo con dejar al cuidado de un familiar, contrastando con un $8 \%$ equivalente a 1 persona deja el cuidado a la guardería.

Esto evidencia que el bienestar integral en este tema, recae en el cónyuge o pareja para el cuidado médico y/o escolar. 


\section{Pregunta 11}

\section{Gráfico 16}

¿Considera usted que la paternidad/maternidad puedan afectar a su crecimiento profesional en la empresa?

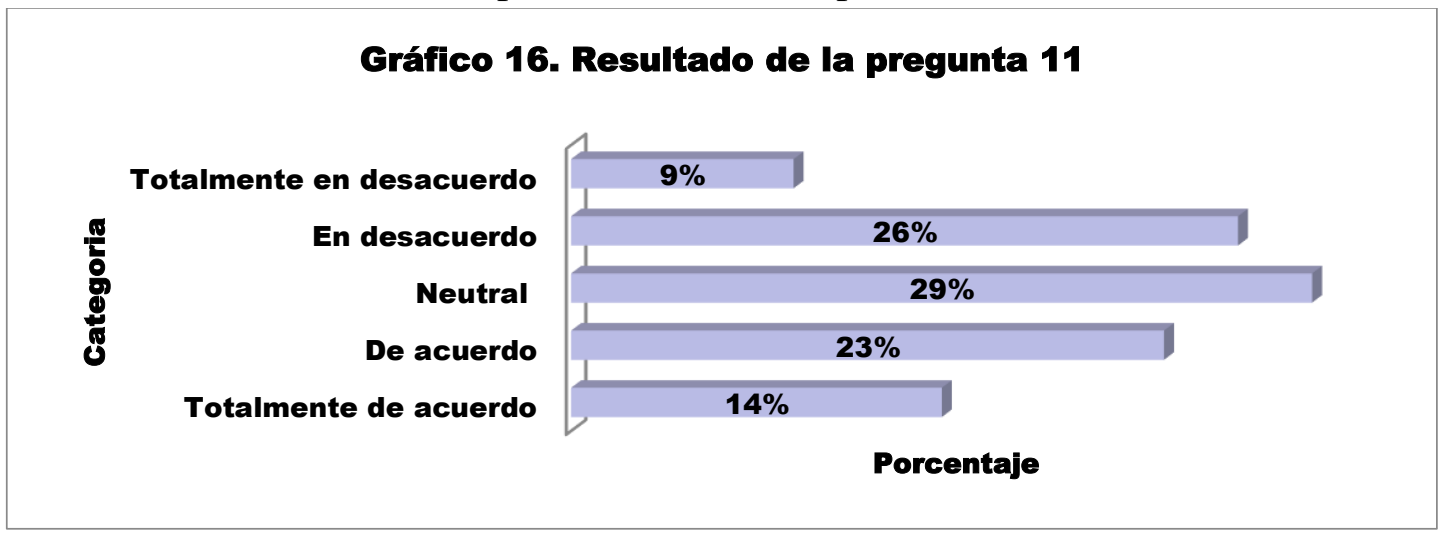

\section{Gráfico 16. Alfa publicaciones}

Fuente: Elaboración propia.

Se puede observar que en la pregunta, sobre si creían los trabajadores encuestados sobre si afectaría el tema de la maternidad/paternidad a la oportunidad de crecer profesionalmente, se obtuvo que, un $29 \%$ equivalente a 10 personas de la muestra se mostraron neutrales frente a esta posibilidad, un $26 \%$ conformado por 9 personas están en desacuerdo con esta deducción, un $23 \%$ equivalente a 8 personas se muestran de acuerdo con la premisa, con un $14 \%$ conformado por 5 personas se encuentran totalmente de acuerdo contrastando con un 9\% equivalente a 3 personas que se muestran en total desacuerdo en cuanto a esta pregunta.

\section{Pregunta 12}

\section{Gráfico 17}

¿Indique que aspectos considera como más importantes para la mejora de las prácticas actuales de la empresa para la conciliación, vida laboral y familiar?

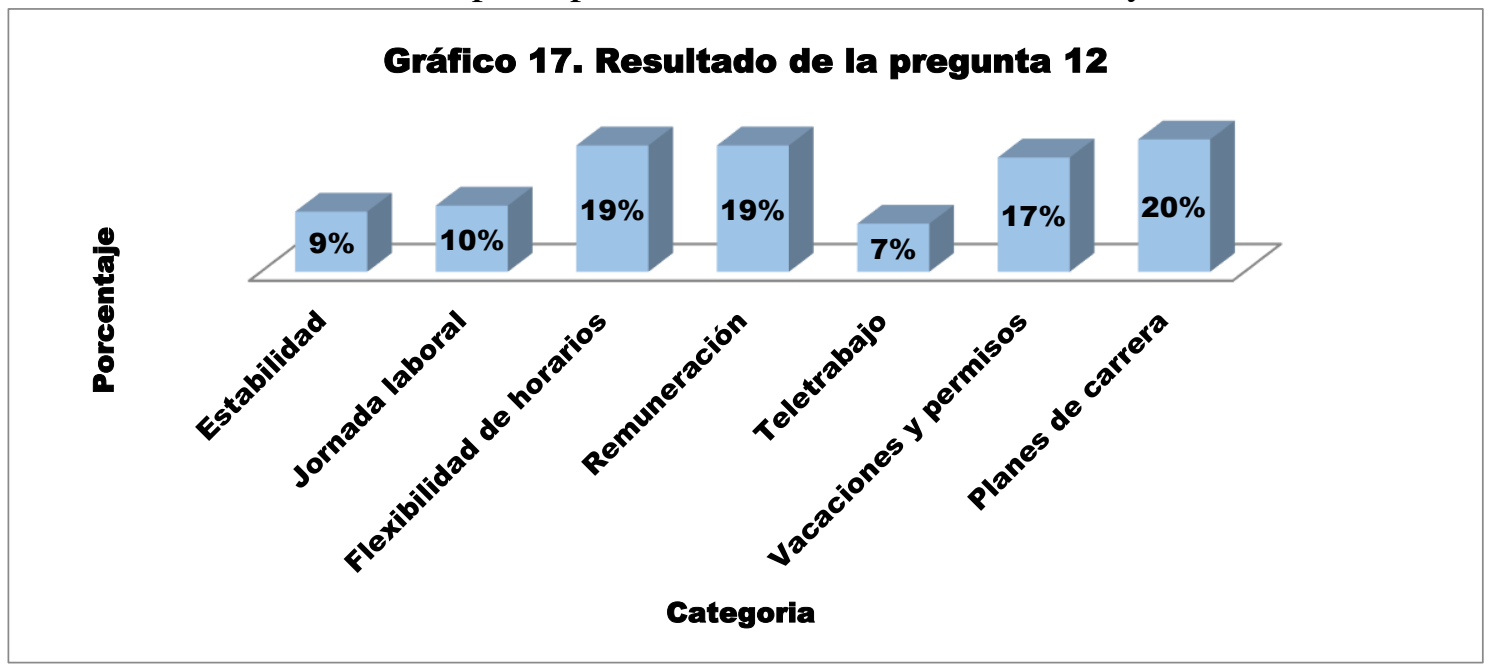

Gráfico 17. Alfa publicaciones

Fuente: Elaboración propia. 
Por lo tanto, se puede determinar los aspectos que consideran las personas encuestadas como prácticas que debería tener la empresa para encontrar un equilibrio en el aspecto vida laboral y familiar. Entre los porcentajes más altos se encuentran con un $20 \%$ el generar planes de carrera junto a un $19 \%$ se encuentran el tener flexibilidad de horarios junto a una remuneración acorde a las bandas salariales del mercado, con un $17 \%$ generar prácticas que beneficien a las personas con respecto al tema de vacaciones y permisos.

En base a las respuestas obtenidas, se establecerá a corto plazo un programa que será presentado a la presidencia y gerencia general para llegar a acuerdos y lograr en los trabajadores de la empresa un equilibrio entre la vida laboral y familiar.

\section{Conclusiones.}

- Hay que recalcar que la empresa al momento, no dispone de programas que fomenten el equilibrio de la vida laboral y familiar de su personal, lo que dispone son de prácticas puntuales que están detalladas en el marco legal y laboral del país.

- Al tener prácticas que fomenten el equilibrio de vida laboral y familiar se fortalecerían los valores de la empresa, mejoraría el clima laboral y se obtendría mayor fidelidad de los colaboradores. Todo esto sería clave para una gestión positiva de talento humano en la empresa.

- La empresa con los resultados obtenidos, fomentará la equidad interna y externa al momento de la contratación de personal, sin discriminación de géneros. Otro aspecto a resaltar es el nivel alto de escolaridad que posee actualmente la empresa.

- Se han identificado buenas prácticas en la concesión de permisos para el personal, tales como otorgar permisos de máximo 4 horas dentro de la jornada para que puedan atender asuntos de tipo personales como familiares, hay que mencionar que dichos permisos deben ser recuperados, no obstante, no se niegan los mismos al personal que lo necesita en las horas y días solicitados.

- Las prácticas que se deberían fortalecerse en la empresa van enfocadas a la flexibilidad de horarios, teletrabajo, remuneraciones, planes de carrera, capacitación, junto a las vacaciones a tiempo y permisos especiales de tipo personal y familiar.

- Finalmente, talento humano de la compañía realizará estas recomendaciones a la Dirección para ser implementadas en el mediano plazo.

- Revisión de bandas salariales acorde al giro del negocio, para obtener equidad interna y externa.

- Establecer planes de carrera objetivos

- Flexibilidad en la otorgación de periodos de vacaciones

- Permisos sin remuneración

- Programas sociales y culturales que integren a la familia con el trabajo

- Permisos adicionales por defunción, enfermedad, maternidad o paternidad. 
- Bonos por nacimientos de hijos, defunciones de familiares hasta el segundo grado de consanguineidad como afinidad.

- Becas para estudios

- Capacitación, entrenamiento y educación mejor focalizada para el personal

- Implementar otras prácticas sobre el salario emocional

- Que la Responsabilidad Social Corporativa RSC, empiece a gestionarse desde el talento humano de la empresa.

\section{Referencias bibliográficas.}

Albizu, E. y Landeta, J. (2011). Dirección estratégica de los recursos humanos. Madrid, España: Ediciones Pirámide.

Deloitte University Press (2015). La Responsabilidad Corporativa; factor estratégico para el crecimiento económico. Recuperado el 14 de Mayo de 2018, de file:///C:/Users/USUARIO/Downloads/RSC\%20informe\%20Deloitte\%20(1).pdf

Ena, B. y Delgado, S. (2012). Recursos Humanos y Responsabilidad Social Corporativa. (1 $\quad$ Ed). Madrid, España: Ediciones Paraninfo

Gómez, M. (2006). Introducción a la metodología de la investigación científica. Córdova. Argentina: Bruja

González, M., \& Olivares, S. (2014). Planeación e integración de los recursos humanos: Capital humano (2a. ed.).

Hernández, R., Fernández, C. y Baptista, P. (2006). Metodología de la Investigación. (4ta Ed.). México, México DF: Mc Graw Hill.

Paniagua, E. (2012). Recursos Humanos y dirección de equipos en restauración. Madrid: Paraninfo.

Revista Líderes. (2017). El salario emocional tiene más importancia https://www.revistalideres.ec/lideres/salario-emocional-importancia productividad.html

Rivas, M. (2014). Gestión del talento humano. Madrid: ESIC.

Santillán,W.(2017). Nuevas prácticas para mejorar el clima laboral. Quito: Don Bosco.

Santillan, W., Ramos, C. y Jadán, J. (2017). Atraer, motivar y retener talentos, la gran misión de los gestores de talento humano. Quito: Don Bosco (ISBN: 978-99429916-5-2).

Viscarra Flores, J. J. (2021). Relación entre el clima laboral y la congruencia de valores en el Centro de Educación Continua (CEC) sede Escuela Politécnica Nacional. 83 hojas. Quito : EPN. 
Werther, W., Keith, D. (2000). Administración de Personal y Recursos Humanos. México: Quinta edición. Mc Graw Hill. 48-225

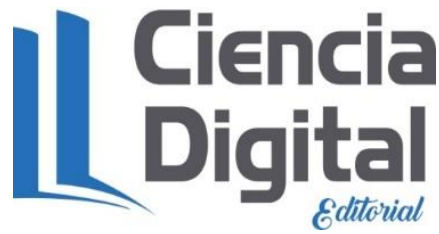




\section{PARA CITAR EL ARTÍCULO INDEXADO.}

Sanguña Carrera, K. L., \& Santillán Marroquín, W. E. . (2021). Propuesta para conciliar el trabajo y la familia en una compañía del sector automotriz. AlfaPublicaciones, 3(3), 6-22. https://doi.org/10.33262/ap.v3i3.64

El artículo que se publica es de exclusiva responsabilidad de los autores y no necesariamente reflejan el pensamiento de la Revista Alfa Publicaciones.

El artículo queda en propiedad de la revista y, por tanto, su publicación parcial y/o total en otro medio tiene que ser autorizado por el director de la Revista Alfa Publicaciones.
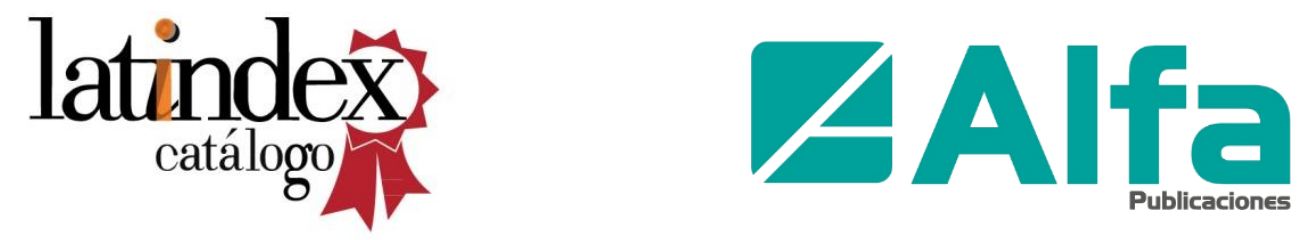